Bull. Austral. Math. Soc.

$46 \mathrm{~B} 20,46 \mathrm{~B} 10,46 \mathrm{~T} 20,46 \mathrm{G} 05$

VOL. 63 (2001) [93-100]

\title{
RESTRICTED WEAK UPPER SEMICONTINUOUS DIFFERENTIALS OF CONVEX FUNCTIONS
}

\author{
Julio Benítez and Vicente Montesinos
}

We characterise restricted weak upper semicontinuity of the subdifferential of convex functions in terms of the Fenchel biconjugate mapping.

\section{INTRODUCTION.}

Given a convex lower semicontinuous function $f$ defined on a real Banach space $X$, the subdifferential of $f$ at $x \in X$ is defined by

$$
\partial f(x):=\left\{x^{*} \in X^{*}:\left\langle y-x, x^{*}\right\rangle \leqslant f(y)-f(x), \forall y \in X\right\},
$$

if $x \in \operatorname{dom}(f)$, while $\partial f(x)=\emptyset$ if $x \in X \backslash \operatorname{dom}(f)$.

A set-valued mapping $\Phi$ from a topological space $\left(X, \tau^{\prime}\right)$ into subsets of another topological space $(Y, \tau)$ is said to be $\left[\tau^{\prime}-\tau\right]$-upper semicontinuous at $x \in X$ if given a $\tau$-open subset $W$ of $Y$ such that $\Phi(x) \subset W$, there exists a $\tau^{\prime}$-neighbourhood $U$ of $x$ such that $\Phi(U) \subset W$. In this paper we shall always consider $X$ a real Banach space endowed with the norm topology and $Y=X^{*}$ endowed with a topology $\tau$. We shall write $\tau$-upper semicontinuous instead of $[\|\cdot\|-\tau]$-upper semicontinuous.

Given a convex function $f$ on an open subset $D$ of a Banach space $X$ and a point of continuity $x_{0} \in D$ of $f$, it can be proved that $\partial f\left(x_{0}\right)$ is a nonempty, $w^{*}$-compact and convex subset of $X^{*}$, and the mapping $x \mapsto \partial f(x)$ is $w^{*}$-upper semicontinuous at $x_{0}$.

Gâteaux differentiability and Fréchet differentiability can be characterised in terms of the continuity of the subdifferential mapping: Given a continuous convex function $f$ on an open subset $D$ of a Banach space $X$ and a point $x_{0} \in D, f$ is Gâteaux differentiable at $x_{0} \in A$ if and only if $\partial f(x)$ is a singleton, and $f$ is Fréchet differentiable at $x_{0}$ if and only if $\partial f(x)$ is a singleton and the subdifferential mapping $x \mapsto \partial f(x)$ is \| * |-upper semicontinuous at $x_{0}$ (for these and related concepts see, for example, [11]).

If the one sided limit in the definition of the derivative of $f$ at a point $x_{0}$ is uniform in every direction, we get a weaker concept than Fréchet differentiability. More precisely,

Received 4th April, 2000

The second named author has been supported in part by DGICYT PB96-0758.

Copyright Clearance Centre, Inc. Serial-fee code: 0004-9727/01 \$A2.00+0.00. 
given a continuous function $f$ defined on an open subset $D$ of a Banach space $X$, we say (following $[5,8]$ ) that $f$ is strongly subdifferentiable at $x_{0} \in D$ if

$$
d^{+} f_{x_{0}}(u):=\lim _{t \rightarrow 0+}\left(f\left(x_{0}+t u\right)-f\left(x_{0}\right)\right) / t
$$

is uniform in $\|u\|=1$. This non-smooth extension of Fréchet differentiability has found several applications (see for example, $[1,5,6,9,10]$ ).

The following definition was introduced in [6]: A set-valued mapping $\Phi$ from a Banach space $X$ into the subsets of $X^{*}$ endowed with the topology $\tau$ is said to be restricted $\tau$-upper semicontinuous at $x \in X$ if given a $\tau$-neighbourhood $W$ of 0 in $X^{*}$ there exists an open neighbourhood $U$ of $x$ in $X$ such that $\Phi(U) \subset \Phi(x)+W$.

In [9] it was proved that given a continuous convex function $f$ defined on an open subset $D$ of a Banach space $X, f$ is strongly subdifferentiable at $x_{0} \in D$ if and only if $\partial f$ is restricted $\|\cdot\|$-upper semicontinuous at $x_{0}$.

In this note we provide, in the spirit of [6], a characterisation of restricted $w$-upper semicontinuity of the subdifferential mapping of a convex function by using the Fenchel biconjugate mapping. Notice that a partial characterisation was obtained in [6] for the duality mapping (that is, $x \mapsto \partial\|\cdot\|(x)$ ). For the use of the concept of restricted $w$-upper semicontinuity of the subdifferential mapping in questions related to the Asplundness and reflexivity of a Banach space we refer to $[2,4,6,7]$ and references therein.

Given a continuous convex function $f$ on an open convex subset $D$ of a Banach space $X$, we can extend $f$ to a lower semicontinuous convex function on $X$, denoted again by $f$, by defining

$$
f(x):=\left\{\begin{array}{cc}
\underset{y \rightarrow x}{\liminf } f(y) & \text { for } x \in \bar{D} \\
+\infty & \text { otherwise. }
\end{array}\right.
$$

Given a convex, proper, lower semicontinuous function $f: X \rightarrow \mathbb{R} \cup\{+\infty\}$, the Fenchel conjugate of $f$ is defined by

$$
f^{*}\left(x^{*}\right):=\sup \left\{\left\langle x, x^{*}\right\rangle-f(x): x \in X\right\} .
$$

Now $f^{*}$ is again convex, proper and lower semicontinuous (in fact, lower $w^{*}$-semicontinuous). Obviously $\left\langle x, x^{*}\right\rangle \leqslant f(x)+f^{*}\left(x^{*}\right)$ for all $x \in X, x^{*} \in X^{*}$ (and the inequality becomes equality if and only if $x^{*} \in \partial f(x)$ ). Moreover, if $\varepsilon \geqslant 0$, then $x^{*} \in \partial_{\varepsilon} f(x)$ if and only if $f(x)+f^{*}\left(x^{*}\right) \leqslant\left\langle x, x^{*}\right\rangle+\varepsilon$ (where $\partial_{\varepsilon} f$ denotes the $\varepsilon$-subdifferential). Also, $\cdot f^{* *} \mid x=f($ see $[3,11])$.

\section{Preliminary RESUlts.}

We shall need the following results: 
THEOREM 2.1. (Brøndsted-Rockafellar) Suppose that $f$ is a convex proper lower semicontinuous function on the Banach space $X$. Then given any point $x_{0} \in \operatorname{dom}(f)$, $\varepsilon>0$ and any $x_{0}^{*} \in \partial_{\varepsilon} f\left(x_{0}\right)$, there exists $x_{\varepsilon} \in \operatorname{dom}(f)$ and $x_{\varepsilon}^{*} \in X^{*}$ such that

$$
x_{\varepsilon}^{*} \in \partial f\left(x_{\varepsilon}\right), \quad\left\|x_{\varepsilon}-x_{0}\right\| \leqslant \sqrt{\varepsilon}, \quad\left\|x_{\varepsilon}^{*}-x_{0}^{*}\right\| \leqslant \sqrt{\varepsilon} .
$$

The Brøndsted-Rockafellar Theorem, together with the local boundedness of the subdifferential mapping at a point of continuity $x_{0}$, allows us to interweave the $\varepsilon$ subdifferential at $x_{0}$ and the subdifferential at a neighbourhood of $x_{0}$. The precise relationship is formulated in the next result:

LEMMA 2.2. Let $f: X \rightarrow \mathbb{R} \cup\{+\infty\}$ be a proper lower semicontinuous convex function. Let $x_{0}$ be a point of continuity of $f$. Then for all $\varepsilon>0$ there exists $\delta>0$ such that

$$
\partial f\left[B\left(x_{0} ; \delta\right)\right] \subset \partial_{\varepsilon} f\left(x_{0}\right) \subset \partial f\left[B\left(x_{0} ; \sqrt{\varepsilon}\right)\right]+\sqrt{\varepsilon} B_{X} \cdot
$$

Proof: $\partial f$ is locally bounded at $x_{0}$, that is, there exists $M>0$ and $N\left(x_{0}\right), a$ neighbourhood of $x_{0}$, such that

$$
\left\|x^{*}\right\| \leqslant M, \quad \forall x^{*} \in \partial f(x), \quad \forall x \in N\left(x_{0}\right) .
$$

Given $\varepsilon>0$, choose $\delta>0$ such that

$$
B\left(x_{0} ; \delta\right) \subset N\left(x_{0}\right), \quad M \delta<\frac{\varepsilon}{2}, \quad\left|f(x)-f\left(x_{0}\right)\right|<\frac{\varepsilon}{2}, \forall x \in B\left(x_{0} ; \delta\right) .
$$

Let $x^{*} \in \partial f\left[B\left(x_{0} ; \delta\right)\right]$, say $x^{*} \in \partial f(x)$ for some $x \in B\left(x_{0} ; \delta\right)$. Then

$$
\begin{aligned}
\left\langle y-x_{0}, x^{*}\right\rangle & =\left\langle y-x, x^{*}\right\rangle+\left\langle x-x_{0}, x^{*}\right\rangle \\
& \leqslant f(y)-f(x)+\left\|x^{*}\right\|\left\|x-x_{0}\right\|<f(y)-f\left(x_{0}\right)+\left|f\left(x_{0}\right)-f(x)\right|+M \delta \\
& <f(y)-f\left(x_{0}\right)+\frac{\varepsilon}{2}+\frac{\varepsilon}{2}=f(y)-f\left(x_{0}\right)+\varepsilon,
\end{aligned}
$$

hence $x^{*} \in \partial_{\varepsilon} f\left(x_{0}\right)$.

The second inclusion is the Brøndsted-Rockafellar Theorem, and does not need the continuity of $f$ at $x_{0}$.

The following proposition can be found in [11]:

Proposition 2.3. Let $f: D \rightarrow \mathbf{R}$ be a convex function on $D$ (a non-empty open and convex subset of $X$ ), continuous at $x_{0} \in D$. Then, for all $y \in X$,

$$
d^{+} f_{x_{0}}(y)=\sup \left\{\left\langle y, x^{*}\right\rangle: x^{*} \in \partial f\left(x_{0}\right)\right\}
$$

and this supremum is attained at some point $x^{*} \in \partial f\left(x_{0}\right)$.

Proposition 2.4. Let $f: X \rightarrow \mathbf{R} \cup\{+\infty\}$ be a convex, proper and lower semicontinuous function. Then epi $\left(f^{* *}\right)=\overline{\operatorname{epi}(f)}^{w^{*}}$. 
Proof: First, assume $f \geqslant 0$. The inclusion $\overline{\operatorname{epi}(f)}^{w^{*}} \subset$ epi $\left(f^{* *}\right)$ follows from $\operatorname{epi}(f) \subset \operatorname{epi}\left(f^{* *}\right)$ and the lower $w^{*}$-semicontinuity of $f^{* *}$. Let $\left(x_{0}^{* *}, \lambda_{0}\right) \in \operatorname{epi}\left(f^{* *}\right)$. Suppose that $\left(x_{0}^{* *}, \lambda_{0}\right) \notin \overline{\operatorname{epi}(f)}^{w^{*}}$. By the Hahn-Banach Theorem, there are $x_{0}^{*} \in X^{*}$, $k, \alpha, \beta \in \mathbb{R}$ such that:

$$
\left\langle x_{0}^{* *}, x_{0}^{*}\right\rangle+k \lambda_{0}<\alpha<\beta<\left\langle x^{* *}, x_{0}^{*}\right\rangle+k \lambda, \quad \forall\left(x^{* *}, \lambda\right) \in \overline{\operatorname{epi}(f)}^{w^{*}} .
$$

From (1) we get $k \geqslant 0$ (if $k<0$, it is enough to take $x \in \operatorname{dom}(f)$ and $\lambda \rightarrow+\infty$ in order to obtain a contradiction). In particular, from (1), we get $\left\langle x, x_{0}^{*}\right\rangle+k f(x)>\beta$, for all $x \in \operatorname{dom}(f)$. Take $\varepsilon>0$. Since $f \geqslant 0$, we get

$$
\left\langle x,-\frac{x_{0}^{*}}{k+\varepsilon}\right\rangle-f(x)<-\frac{\beta}{k+\varepsilon}, \quad \forall x \in \operatorname{dom}(f),
$$

hence $f^{*}\left(-x_{0}^{*} /(k+\varepsilon)\right) \leqslant-\beta /(k+\varepsilon)$. Then

$$
\begin{aligned}
f^{* *}\left(x_{0}^{* *}\right) & \geqslant\left\langle x_{0}^{* *},-\frac{x_{0}^{*}}{k+\varepsilon}\right\rangle-f^{*}\left(-\frac{x_{0}^{*}}{k+\varepsilon}\right) \\
& \geqslant\left\langle x_{0}^{* *},-\frac{x_{0}^{*}}{k+\varepsilon}\right\rangle+\frac{\beta}{k+\varepsilon}=\frac{1}{k+\varepsilon}\left[\beta-\left\langle x_{0}^{* *}, x_{0}^{*}\right\rangle\right]>\frac{\beta-\alpha+k \lambda_{0}}{k+\varepsilon} .
\end{aligned}
$$

If $k=0$, then $f^{* *}\left(x_{0}^{* *}\right)>(\beta-\alpha) / \varepsilon$. As $\varepsilon>0$ was arbitrary, we get $x_{0}^{* *} \notin \operatorname{dom}\left(f^{* *}\right)$, a contradiction. If $k \neq 0$, since $\varepsilon>0$ was arbitrary, we get $f^{* *}\left(x_{0}^{* *}\right) \geqslant\left(\beta-\alpha+k \lambda_{0}\right) / k>\lambda_{0}$. This contradicts $\left(x_{0}^{* *}, \lambda_{0}\right) \in \operatorname{epi}\left(f^{* *}\right)$.

Now, if $f: X \rightarrow \mathbb{R} \cup\{+\infty\}$ is an arbitrary proper semicontinuous convex function, choose $x_{0}^{*} \in \operatorname{dom}\left(f^{*}\right)$. Consider $g: X \rightarrow \mathbb{R} \cup\{+\infty\}$ given by $g(x)=f(x)+f^{*}\left(x_{0}^{*}\right)-\left\langle x, x_{0}^{*}\right\rangle$. This function, obviously, is proper, lower semicontinuous and convex. Moreover $\operatorname{dom}(f)=$ $\operatorname{dom}(g)$ and $g \geqslant 0$. Now, a simple calculation shows $g^{* *}\left(x^{* *}\right)=f^{* *}\left(x^{* *}\right)+f^{*}\left(x_{0}^{*}\right)-\left\langle x^{* *}, x_{0}^{*}\right\rangle$ for all $x^{* *} \in X^{* *}$. By the first part of the proof, the proposition holds for $g$, and hence for $f$.

\section{REMARKS.}

1. Note that Goldstine's Theorem is a particular case of the former proposition: It is enough to take as $f$ the indicator function $\delta_{B_{X}}$ of the closed unit ball of $X$ (that is, $\delta_{B_{X}}(x)=0$ if $\|x\| \leqslant 1, \delta_{B_{X}}(x)=+\infty$ if $\|x\|>1$ ), a proper lower semicontinuous convex function. Obviously, $f^{*}$ is the dual norm. Let $x^{* *} \in B_{X} \ldots$. As

$$
f^{* *}\left(x^{* *}\right)=\sup \left\{\left\langle x^{* *}, x^{*}\right\rangle-\left\|x^{*}\right\|: x^{*} \in X^{*}\right\} \leqslant 0<+\infty,
$$

$\underset{\bar{B}_{X}}{\text { we }} \underset{w^{*}}{\text { get }} x^{* *} \in \operatorname{dom}\left(f^{* *}\right)$. By Proposition 2.4, $\operatorname{dom}\left(f^{* *}\right)=\overline{\operatorname{dom}(f)} w^{*}=$ $\overline{B_{X}} w^{*}$.

2. This proposition gives a description of $f^{* *}$, sometimes simpler than the original one. 
CoRollary 2.5. Let $f: X \rightarrow \mathbb{R} \cup\{+\infty\}$ be a proper lower semicontinuous convex function. Then, given $x_{0} \in X$,

1. $\partial f\left(x_{0}\right)=\partial f^{* *}\left(x_{0}\right) \cap X^{*}$.

2. If $f$ is continuous at $x_{0}, f^{* *}$ is also continuous at $x_{0}$.

Proof: (1) is a consequence of two well-known facts (see [11]): $f^{* *}$ induces $f$ on $X$, and $x_{0}^{*} \in \partial f\left(x_{0}\right)$ if and only if $\left\langle x_{0}, x_{0}^{*}\right\rangle=f\left(x_{0}\right)+f^{*}\left(x_{0}^{*}\right)$.

To prove (2), assume $f$ (but not $f^{* *}$ ) is continuous at $x_{0}$. Let $\mathcal{N}$ be a basis of $w^{*}$ open neighbourhoods of 0 in $X^{* *}$. Then there exists $\varepsilon>0$ and $x_{N}^{* *} \in x_{0}+N, N \in \mathcal{N}$, such that $\left|f^{* *}\left(x_{N}^{* *}\right)-f\left(x_{0}\right)\right| \geqslant \varepsilon$. As $\overline{\operatorname{epi}(f)}{ }^{*^{*}}=\operatorname{epi}\left(f^{* *}\right)$ and $f^{* *}$ is lower semicontinuous, it is possible to choose $x_{N} \in\left(x_{0}+N\right) \cap X$ such that $f^{* *}\left(x_{N}^{* *}\right) \leqslant f\left(x_{N}\right)<f^{* *}\left(x_{N}^{* *}\right)+\varepsilon / 2$, $N \in \mathcal{N}$. It follows that $x_{N} \stackrel{w}{\rightarrow} x_{0}$ and $\left|f\left(x_{N}\right)-f\left(x_{0}\right)\right| \geqslant \varepsilon / 2$, a contradiction.

COROLlary 2.6. Let $f: X \rightarrow \mathbf{R} \cup\{+\infty\}$ be a proper lower semicontinuous convex function. Then, given $x_{0}^{* *} \in \operatorname{dom}\left(f^{* *}\right)$,

$$
f^{* *}\left(x_{0}^{* *}\right)=\inf \left\{\underset{i}{\liminf } f\left(x_{i}\right): x_{i} \subset \operatorname{dom}(f), x_{i} \stackrel{w^{*}}{\rightarrow} x_{0}^{* *}\right\}
$$

Proof: By Proposition 2.4 it is obvious that $\operatorname{dom}\left(f^{* *}\right)=\overline{\operatorname{dom}(f)}{ }^{w^{*}}$. Now, given a net $\left(x_{i}\right)_{i \in I} \subset \operatorname{dom}(f), x_{i} \stackrel{w^{*}}{\rightarrow} x_{0}^{* *}$, by the $w^{*}$-lower semicontinuity of $f^{* *}$ we get $f^{* *}\left(x_{0}^{* *}\right) \leqslant$ liminf $f\left(x_{i}\right)$. On the other hand, again by Proposition 2.4, given $\varepsilon>0$ we can find a net $\left(x_{i}\right)_{i \in I} \subset \operatorname{dom}(f)$ and $\lambda_{i} \in \mathbb{R}$ such that $x_{i} \stackrel{w^{*}}{\rightarrow} x_{0}^{* *},\left(x_{i}, \lambda_{i}\right) \in \operatorname{epi}(f)$ and $\lambda_{i}<f^{* *}\left(x_{0}^{* *}\right)+\varepsilon$. As $f\left(x_{i}\right) \leqslant \lambda_{i}, i \in I$, we get the conclusion.

COROLlary 2.7. Let $f: X \rightarrow \mathbb{R} \cup\{+\infty\}$ be a proper lower semicontinuous convex function. Then, given $x_{0} \in \operatorname{dom}(f)$ and $\varepsilon>0$,

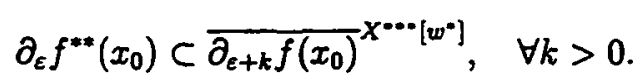

ProOF: Let $x^{* * *} \in \partial_{\varepsilon} f^{* *}\left(x_{0}\right)$. Then $f^{* *}\left(x_{0}\right)+f^{* * *}\left(x^{* * *}\right) \leqslant\left\langle x_{0}, x^{* * *}\right\rangle+\varepsilon$. It follows that

$$
f^{* * *}\left(x^{* * *}\right) \leqslant\left\langle x_{0}, x^{* * *}\right\rangle-f^{* *}\left(x_{0}\right)+\varepsilon<\left\langle x_{0}, x^{* * *}\right\rangle-f^{* *}\left(x_{0}\right)+\varepsilon+k / 2 .
$$

By the previous corollary, there exists a net $\left(x_{i}^{*}\right)_{i \in I}$ in $X^{*}$ such that $x_{i}^{*} \rightarrow x^{* * *}$ in $X^{* * *}\left[w^{*}\right]$, $f^{*}\left(x_{i}^{*}\right)<\left\langle x_{0}, x^{* * *}\right\rangle-f^{* *}\left(x_{0}\right)+\varepsilon+k / 2, \forall i \in I$ and $\left|\left\langle x_{0}, x_{i}^{*}-x^{* * *}\right\rangle\right|<k / 2$. We get

$$
f\left(x_{0}\right)+f^{*}\left(x_{i}^{*}\right)<\left\langle x_{0}, x^{* * *}\right\rangle+\varepsilon+\frac{k}{2}<\left\langle x_{0}, x_{i}^{*}\right\rangle+\varepsilon+k .
$$

Then, $x_{i}^{*} \in \partial_{\varepsilon+k} f\left(x_{0}\right), \forall i \in I$. The conclusion follows.

\section{A CHARACTERISATION OF THE RESTRICTED $w$-UPPER SEMICONTINUITY.}

If $x \in X$ and $\delta>0$, we shall denote by $B^{* *}\left(x_{0} ; \delta\right)$ the open ball in $X^{* *}$ of radius $\delta$ and centred at $x_{0}$.

Now we are ready to prove the main result in this note: 
THEOREM 3.1. Let $f: X \rightarrow \mathbf{R} \cup\{+\infty\}$ be a proper lower semicontinuous convex function. Let $x_{0}$ be a point of continuity of $f$. Then the following assertions are equivalent:

1. $\partial f$ is restricted $w$-upper semicontinuous at $x_{0}$.

2. For all $N$, a w-neighbourhood of 0 in $X^{*}$, there is $\varepsilon>0$ such that $\partial_{\varepsilon} f\left(x_{0}\right) \subset$ $\partial f\left(x_{0}\right)+N$

3. $\partial f\left(x_{0}\right)$ is dense in $\partial f^{* *}\left(x_{0}\right)$ in $X^{* * *}\left[w^{*}\right]$.

4. $d^{+} f_{z_{0}}^{* *}(\cdot)=\sup \left\{\left(\cdot, x^{*}\right): x^{*} \in \partial f\left(x_{0}\right)\right\}$.

Proof: (1) $\Rightarrow(2)$ : Let $N$ be a convex $w$-neighbourhood of 0 in $X^{*}$. By hypothesis there is $\delta>0$ such that $\partial f\left[B\left(x_{0} ; \delta\right)\right] \subset \partial f\left(x_{0}\right)+N / 2, \delta B_{X} \cdot \subset N / 2$. Now, by Lemma 2.2 ,

$$
\partial_{\delta^{2}} f\left(x_{0}\right) \subset \partial f\left[B\left(x_{0} ; \delta\right)\right]+\delta B_{X} \cdot \subset \partial f\left(x_{0}\right)+\frac{1}{2} N+\frac{1}{2} N \subset \partial f\left(x_{0}\right)+N .
$$

It is enough to choose $\varepsilon=\delta^{2}$.

$(2) \Rightarrow(3)$ : Given a closed neighbourhood $N^{* *}$ of 0 in $X^{* * *}\left[w^{*}\right]$, let $\varepsilon>0$ be as in (2). Then, using Corollary 2.5, Corollary 2.7 and the fact that $\frac{w^{*} f\left(x_{0}\right)}{X^{\cdots \cdot}\left[w^{*}\right]}$ is compact and $N:=N^{* *} \cup X^{*}$ is closed in $X^{* *}\left[w^{*}\right]$,

$$
\begin{aligned}
& \partial f\left(x_{0}\right) \subset \partial f^{* *}\left(x_{0}\right) \subset \partial_{\varepsilon / 2} f^{* *}\left(x_{0}\right) \subset{\overline{\partial_{\varepsilon} f\left(x_{0}\right)}}^{\left.X \cdots \cdot \omega^{*}\right]} \\
& \subset{\overline{\partial f\left(x_{0}\right)+N}}^{x^{\cdots \cdot}\left[w^{*}\right]} \subset{\overline{\partial f\left(x_{0}\right)}}^{X^{\cdots}{ }^{\left[w^{*}\right]}+N^{* *}} \text {. }
\end{aligned}
$$

This proves (3).

(3) $\Rightarrow(1)$ : Let $N$ a $w$-neighbourhood of 0 in $X^{*}$. Take a convex $w^{*}$-neighbourhood of 0 in $X^{* *}, N^{*}$, such that $N^{*} \cap X^{*} \subset N$. By Corollary 2.5, $f^{* *}$ is continuous at $x$, so $\partial f^{* *}$ is upper $w^{*}$-semicontinuous at $x$. Hence there exists $\delta>0$ such that

$$
\partial f^{* *}\left(B^{* *}(x ; \delta)\right) \subset \partial f^{* *}(x)+N^{*} / 2
$$

By hypothesis, $\partial f^{* *}(x) \subset \partial f(x)+N^{*} / 2$. It follows that

$$
\partial f^{* *}\left(B^{* *}(x ; \delta)\right) \subset \partial f^{* *}(x)+N^{*} / 2 \subset \partial f(x)+N^{*} / 2+N^{*} / 2 \subset \partial f(x)+N^{*} .
$$

It is now enough to use Corollary 2.5 to get $\partial f(B(x ; \delta)) \subset \partial f(x)+N$.

$(3) \Leftrightarrow(4)$ : By Proposition 2.3 and Corollary 2.5,

$$
d^{+} f_{x_{0}}^{* *}(\cdot)=\sup \left\{\left\langle\cdot, x^{* * * *}\right\rangle: x^{* * *} \in \partial f^{* * *}\left(x_{0}\right)\right\} .
$$

Now, using the Hahn-Banach Theorem, the equivalence is obvious.

Note that the equivalence (1) $\Leftrightarrow(2)$ is valid not only for restricted $w$-upper semicontinuity, but also for $\tau$-upper semicontinuity, $\tau$ a Hausdorff topology weaker than the norm-topology. More precisely: 
THEOREM 3.2. Let $f: X \rightarrow \mathbf{R} \cup\{+\infty\}$ be a proper lower semicontinuous convex function. Let $x_{0} \in X$ be a point of continuity of $f$. If $\tau$ is a topology on $X^{*}$ weaker than the norm topology, then the following assertions are equivalent:

1. $\partial f$ is restricted $\tau$-upper semicontinuous at $x_{0}$.

2. For every $\tau$-neighbourhood $N$ of 0 in $X^{*}$, there is $\varepsilon>0$ such that $\partial_{\varepsilon} f\left(x_{0}\right) \subset$ $\partial f\left(x_{0}\right)+N$.

Proof: For $(1) \Rightarrow(2)$ the same proof used in the previous theorem, $(1) \Rightarrow(2)$, works. To prove $(2) \Rightarrow(1)$, use Lemma 2.2 .

This characterisation can be considered as the analogue of the Śmulyan Test.

It is well known that if the dual norm of $X^{*}$ is locally uniformly rotund, then the norm of $X$ is Fréchet differentiable. The next proposition, that uses the previous theorem, extends this result. Note that the Fenchel conjugate of the norm of a Banach space $X$ is the indicator function of $B_{X}$ -

Proposition 3.3. Let $f: D \rightarrow \mathbb{R}$ be a convex, continuous function defined on $D$, a non-empty open subset of $X$. Let $x_{0} \in D$. If $\tau$ is a Hausdorf topology on $X^{*}$ weaker than the norm topology, then the following assertions are equivalent:

1. $f$ is Gâteaux differentiable at $x_{0}$ and $\partial f$ is restricted $\tau$-upper semicontinuous at $x_{0}$.

2. For every $\tau$-neighbourhood $N$ of 0 in $X^{*}$ and $x^{*} \in \partial f\left(x_{0}\right)$, there exists $\delta=\delta\left(x^{*}, N\right)$ such that

$$
f\left(x_{0}\right)+\frac{1}{2}\left(f^{*}\left(x^{*}\right)+f^{*}\left(y^{*}\right)\right)-\delta<\frac{1}{2}\left\langle x_{0}, x^{*}+y^{*}\right\rangle \Rightarrow y^{*} \in x^{*}+N .
$$

Proof: $(1) \Rightarrow(2)$. Let $N$ be a $\tau$-neighbourhood of 0 in $X^{*}$ and $\left\{x^{*}\right\}=\partial f\left(x_{0}\right)$. By the previous theorem there exists $\varepsilon>0$ such that $\partial_{\varepsilon} f\left(x_{0}\right) \subset x^{*}+N$. Take $y^{*} \in X^{*}$ such that

$$
f\left(x_{0}\right)+\frac{1}{2}\left(f^{*}\left(x^{*}\right)+f^{*}\left(y^{*}\right)\right)-\frac{\varepsilon}{2}<\frac{1}{2}\left\langle x_{0}, x^{*}+y^{*}\right\rangle .
$$

A simple calculation shows that $f\left(x_{0}\right)+f^{*}\left(y^{*}\right)<\left\langle x_{0}, y^{*}\right\rangle+\varepsilon$. It follows that $y^{*} \in$ $\partial_{\varepsilon} f\left(x_{0}\right) \subset x^{*}+N$.

$(2) \Rightarrow(1)$. First, we shall prove that $f$ is Gâteaux differentiable at $x_{0}$. If not, there would exist $x_{1} \neq x_{2}$ in $\partial f\left(x_{0}\right)$. Choose a $\tau$-neighbourhood $N$ of 0 in $X^{*}$ such that $\left(x_{i}^{*}+N\right) \cap\left(x_{2}^{*}+N\right)=\emptyset$. Let $\delta_{i}=\delta_{i}\left(x_{i}^{*}, N\right)$ be as in $(2)(i=1,2)$ and let $\delta:=\min \left\{\delta_{1}, \delta_{2}\right\}$. Take $y^{*} \in \partial_{\delta} f\left(x_{0}\right)$. A simple calculation shows that

$$
f\left(x_{0}\right)+\frac{1}{2}\left(f^{*}\left(x_{i}^{*}\right)+f^{*}\left(y^{*}\right)\right)-\delta_{i}<\frac{1}{2}\left\langle x_{0}, x_{i}^{*}+y^{*}\right\rangle,
$$

for $i=1,2$. By hypothesis, $y^{*} \in\left(x_{1}^{*}+N\right) \cap\left(x_{2}^{*}+N\right)$, a contradiction.

Now, let $N$ be a $\tau$-neighbourhood of 0 in $X^{*}$. Since $f$ is Gâteaux differentiable at $x_{0}, \partial f\left(x_{0}\right)=\left\{x_{0}^{*}\right\}$. Given $N$ and $x_{0}^{*}$, we get $\delta=\delta\left(x_{0}^{*}, N\right)$ as in (2). It is easy to 
prove that $\partial_{\delta} f\left(x_{0}\right) \subset x_{0}^{*}+N=\partial f\left(x_{0}\right)+N$. By Theorem 3.2, $\partial f$ is restricted $\tau$-upper semicontinuous at $x_{0}$.

\section{REFERENCES}

[1] C. Aparicio, F. Ocaña, R. Payá and A. Rodriguez, 'A non-smooth extension of Fréchet differentiability of the norm with applications to numerical ranges', Glasgow Math. J. 28 (1986), 121-137.

[2] J. Benitez and V. Montesinos, 'On restricted weak upper semicontinuous set valued mappings and reflexivity', Bol. Un. Mat. Ital. 8 (1999), 577-583.

[3] H. Brezis, Analyse fonctionelle: Theorie et applications, (Collections of Applied Mathematics for the Masters degree) (Masson et Cie., 1983).

[4] M.D. Contreras and R. Payá, 'On upper semicontinuity of duality mappings', Proc. Amer. Math. Soc. 121 (1994), 451-459.

[5] C. Franchetti and R. Payá, 'Banach spaces with strongly subdifferentiable norm', Bol. Un. Mat. Ital. 7 (1993), 45-70.

[6] J. Giles, D. Gregory and B. Sims, 'Geometrical implications of upper semicontinuity of the duality mapping on a Banach space', Pacific J. Math. 79 (1978), 99-108.

[7] J. Giles and W. B. Moors, 'Generic continuity of restricted weak upper semicontinuous set valued mappings', Set Valued Anal. 4 (1996), 25-39.

[8] J. Giles and S. Sciffer, 'Fréchet directional differentiability and Fréchet differentiability', Comment. Math. Univ. Carolin. 37 (1996), 489-497.

[9] G. Godefroy, Some applications of Simons' inequality, Seminar of Functional Analysis. Vol 1 (Universidad de Murcia, 1987).

[10] G. Godefroy, V. Montesinos and V. Zizler, 'Strong subdifferentiability of norms and geometry of Banach spaces', Comment. Math. Univ. Carolin 36 (1995), 493-502.

[11] R. Phelps, Convex functions, monotone operators and differentiability (2nd ed.), Lecture Notes in Math. 1364 (Springer-Verlag, Berlin, Heidelberg, New York, 1993).

Depto. Matemática Aplicada

(ETSIT), Apartado 22012

E-46071 Valencia

Spain

e-mail: jbenitez@mat.upv.es
Depto. Matemática Aplicada

(ETSIT), Apartado 22012

E-46071 Valencia

Spain

e-mail: vmontesinos@mat.upv.es 